\title{
Vertical Density Representation and Its Applications
}


This page intentionally left blank 


\section{Vertical Density \\ Representation and Its Applications}

\section{Marvin D Troutt}

Kent State University, USA

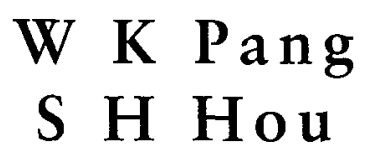

The Hong Kong Polytechnic University, China 


\section{Published by}

World Scientific Publishing Co. Pte. Ltd.

5 Toh Tuck Link, Singapore 596224

USA office: Suite 202, 1060 Main Street, River Edge, NJ 07661

UK office: 57 Shelton Street, Covent Garden, London WC2H 9HE

\section{British Library Cataloguing-in-Publication Data}

A catalogue record for this book is available from the British Library.

\section{VERTICAL DENSITY REPRESENTATION AND ITS APPLICATIONS}

Copyright $\odot 2004$ by World Scientific Publishing Co. Pte. Ltd.

All rights reserved. This book, or parts thereof, may not be reproduced in any form or by any means, electronic or mechanical, including photocopying, recording or any information storage and retrieval system now known or to be invented, without written permission from the Publisher.

For photocopying of material in this volume, please pay a copying fee through the Copyright Clearance Center, Inc., 222 Rosewood Drive, Danvers, MA 01923, USA. In this case permission to photocopy is not required from the publisher.

ISBN 981-238-693-9 


\section{Dedications}

To the memory of my Mother, to my Father, to Mark, Marleen and Andrew, and to Helen.

To my parents, to my wife, Sui-Ching, to Wai-Ying and Chun-Yan.

To my parents and my family.

MDT, WKP, SHH 
This page intentionally left blank 


\section{Preface}

Since the first paper related to Vertical Density Representation (VDR) appeared in 1991 several papers have been published and work continues on the topic. VDR arose in connection with the analysis of performance measurement. Consideration of the Box-Muller method for generation of normal variates led to a question about the density (probability density function, or pdf) of the density function itself. The purpose of this book is to survey these results and provide some new unpublished results. We hope to have made contact with the major published articles on the topic. While it is possible that some could have been missed, we have made a diligent search and have sought the advice of other researchers on the topic.

VDR may be regarded as a special kind of variable transformation but may also be considered as a more general density-modeling tool. By assuming that a variate is uniformly distributed on the contours or level curves of a given function in real n-dimensional space, and considering the density of the ordinate of the given function, the density of the original variate can be represented. Basic results and extensions are discussed. Several applications are described for use with Monte Carlo simulation. The uniformly distributed on contours assumption can be relaxed with what we call the General VDR Theorem. An application of that result is made to a problem in inverse linear programming.

A new result on the analysis of correlation into two distinct components is given along with its potential value in the aggregation of experts. Also a new result on densities of orbit values produced by chaos generators is given along with the construction of a large class of chaos-based uniform random number generators. We also provide a case study in applying VDR 
in connection with what may be called behavioral estimation. VDR considerations are brought to bear in developing a validation technique for the estimation method. Then a further application to estimating benchmark costs and cost matrices is given. Finally in the last chapter, we discuss some future research questions and work in progress for several of the topics discussed in the earlier chapters.

In order to have a good understanding of the book, a background in mathematics and statistics at the graduate level will be best. However, readers having had a calculus-based statistics course and some acquaintance with Lebesgue measure should fare reasonably well. In the past, statistical theory has depended primarily on the Riemann integral. VDR depends more heavily on Lebesgue measure. However, the derivations are kept intuitive with respect to those issues in so far as possible.

We wish to thank Elsevier Science (http://www.elsevier.com), Taylor and Francis (http://www.tandf.co.uk) and the Decision Sciences Institute for granting us permissions to use some of the materials from the papers published in the European Journal of Operational Research, Statistics and Decision Sciences (http://decisionsciences.org) Journal. This monograph is also supported by the research grant committee of the Hong Kong Polytechnic University (Grant code: A-PD05).

We also wish to make a special acknowledgment of Professor Samuel Kotz. Without his early support and contributions to the topic, this book may not have been possible.

Marvin D. Troutt

Graduate School of Management

Kent State University

Kent, Ohio, USA

Wan-Kai Pang and Shui-Hung Hou

Department of Applied Mathematics

The Hong Kong Polytechnic University

Hong Kong, SAR,

China

August 31, 2003 


\section{Contents}

Preface vii

Chapter 1 Vertical Density Representation 1

1.1 Original Motivation . . . . . . . . . . . . . . . 3

1.2 The Density of the Density Ordinate . . . . . . . . . 7

1.2.1 A Formal Definition of Vertical Density Representation (VDR) . . . . . . . . . . . . . . 10

1.3 Elementary Applications of Theorem $1.2 \ldots \ldots \ldots . . . . .11$

1.3.1 VDR as a Kind of Variable Transformation . . . . . . 11

1.4 Construction of Hybrid Densities: Some Univariate Examples . 12

1.5 Further Results in VDR-Type Density Representation . . . . . 14

1.6 Further Remarks on Vertical Density Representation . . . . 17

1.6.1 Nonuniform Contour Densities . . . . . . . . . . . 18

1.7 Vertical Density Representation in the General Case . . . . . 19

1.7.1 Examples . . . . . . . . . . . . . . . . 22

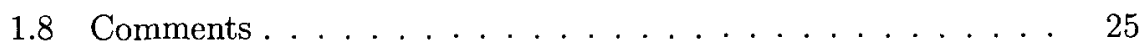

Chapter 2 Applications of Vertical Density Representation 27

2.1 Application I: Ordering of Distributions . . . . . . . . . . . 27

2.2 Standard Measurement in Tail Behaviour Using VDR . . . . 29

2.2 .1 Discussion. . . . . . . . . . . . . . . . . 31

2.3 A New Class of Very Thick-Tailed Densities . . . . . . . . . 32

2.4 Application II . . . . . . . . . . . . . . . . . . 34

2.4 .1 The Univariate Case . . . . . . . . . . . . . . . . . 34

2.5 Vertical and Contour Components of Correlation . . . . . . 36 
2.6 Discussion . . . . . . . . . . . . . . . . . . . . . . . . . . 39

2.7 Further Considerations . . . . . . . . . . . . . . . . . 42

Chapter 3 Multivariate Vertical Density Representation 45

3.1 Multivariate VDR and Conditional Density . . . . . . . . . 46

3.2 Some Results on the Multivariate Normal Distribution . . . . . 49

3.3 An Application of Multivariate VDR . . . . . . . . . . 52

3.4 Tail Behaviour and Multivariate VDR . . . . . . . . 55

3.4.1 Spherically Symmetric Distributions . . . . . . . . . 55

3.4.2 Multivariate Normal Distribution . . . . . . . . . . . 57

3.4.3 Tail Behaviour of the Multivariate Pearson Type II Distribution .................. 60

3.4.4 Tail Behaviour of the Multivariate Spherically Symmetric Logistic Distribution . . . . . . . . . . . . . . . 61

3.4.5 Multivariate Uniform Distribution on the Unit Sphere . 63

3.5 Tail Behavior of $L_{1}$ and $L_{p}$-Norm Symmetric Distributions . . 63

3.5.1 $L_{1}$-Norm Symmetric Distributions . . . . . . . . 63

3.5.2 $L_{p}$-Norm Symmetric Distributions . . . . . . . . 64

3.6 Multivariate Burr, Pareto and Logistic Distributions . . . . . 65

3.7 VDR for the Multivariate Exponential Power Distribution . . . 66

Chapter 4 Applications of Multivariate VDR 69

4.1 Generation of Non-Uniform Random Variates: An Overview . . 69

4.1.1 The Vertical Strip Method . . . . . . . . . . . . 71

4.2 Generation of the Uniform Distribution . . . . . . . . . . . 72

4.3 The Vertical Strip Method . . . . . . . . . . . . . 77

4.3.1 The Geometric Vertical Representation of a Density . . 78

4.3.2 Generation of Random Variates from an Exponential Distribution . . . . . . . . . . . . . . . 80

4.3.3 Generation of Random Variates from a Normal Distribution . . . . . . . . . . . . . . . 84

4.4 Computational Performance . . . . . . . . . . . . 87

4.5 Generation of Multivariate Distributions . . . . . . . . . 89

4.6 Alternative Version of Multivariate VDR . . . . . . . . . . 94

4.7 The Uniform Distribution on a Manifold in $\Re^{n} \ldots \ldots \ldots 98$

4.8 Comments . . . . . . . . . . . . . . . . . . . . . 99

$\begin{array}{lll}\text { Chapter } 5 & \text { VDR and Chaos } & 101\end{array}$ 
5.1 The Relationship between VDR and Chaos . . . . . . . 102

5.2 Recursions and the Vertical Density . . . . . . . . . . . . 104

5.3 The Logistic Chaos Generator . . . . . . . . . . . . . . . . . . . . . . . . . . . . . . . . . . . .

5.4 The Uniform Density . . . . . . . . . . . . . . . . . . 108

5.5 Computations of the Sharkfin Generator . . . . . . . . . . 112

5.6 Generalizations of Sharkfin Generators . . . . . . . . . 116

5.7 Comments . . . . . . . . . . . . . . . . . . . . . . . 119

Chapter 6 Management Science Applications of VDR-I 121

6.1 Tolstoy's Law of the Mode $(\mathrm{TLM}) \ldots \ldots \ldots \ldots . \ldots . . \ldots 122$

6.1.1 A Stochastic Converse Formulation . . . . . . . . . 125

6.1.2 Weak Consensus and Accuracy . . . . . . . . . . . . 131

6.2 Normal-Like Performance on Finite Intervals . . . . . . . . 132

6.2.1 Entropy-Based Generalizations of the Normal Density . 133

6.2 .2 Normal VDR . . . . . . . . . . . . . . 135

6.2.3 Normal-like Performance on $[0,1] \ldots \ldots \ldots$

6.2 .4 Conclusion . . . . . . . . . . . . . . . 138

6.3 Unimodality on the Half-Interval . . . . . . . . . . . . 138

6.3.1 Relationships on the Half-Interval . . . . . . . . 139

6.4 Unimodality . . . . . . . . . . . . . . . . . . . 140

6.5 Strata Shift Densities . . . . . . . . . . . . . . . . 143

6.6 The Use of the General VDR Theorem . . . . . . . . . . . . 144

6.6.1 An Inverse Linear Programming Problem . . . . . . . 144

6.6.2 Comparison to a First Principles Approach . . . . . . . 145

6.7 Conclusion . . . . . . . . . . . . . . 147

Chapter 7 Management Science Applications of VDR-II 149

7.1 The Aggregate Production Planning Problem . . . . . . . . 150

7.2 Minimum Decisional Regret Estimation of Cost Parameters . . 152

7.2.1 Conceptual Model of the Planning Process . . . . . . 152

7.2.2 Some Notational Conventions and Definitions . . . . . 153

7.3 Decisional Regret . . . . . . . . . . . . . . . . . 154

7.3.1 Handling of Costs Assumed to be Known . . . . . . 159

7.4 Validation . . . . . . . . . . . . . . . . 160

7.4.1 The Target-Mode Agreement Criterion . . . . . . . . . 160

7.4.2 Stipulated Model Choice and Routine Validation . . . 163

7.5 Data and Binary Variable Concerns . . . . . . . . . . . . 164

7.5 .1 Data Set Issues . . . . . . . . . . . . . . . . . . . 164 
7.5.2 Other Potential Application Settings . . . . . . . . . 165

7.5.3 Decision Space and Binary Decision Variables . . . . . . 167

7.6 Re-estimation and Continuous Improvement . . . . . . . . . . 168

7.7 Comparisons with Management Coefficients Theory . . . . . 169

7.8 Inappropriate Convergence . . . . . . . . . . . . . . 170

7.9 Miscellaneous Remarks . . . . . . . . . . . . . . . . 172

7.10 Conclusions . . . . . . . . . . . . . . . . 173

Chapter 8 Management Science Applications of VDR-III 179

8.1 The Multiple Driver - Single Cost Pool Case . . . . . . . . 180

8.1.1 The Importance of Cost Benchmarking . . . . . . . . . 182

8.1.2 The Rates Departments Data . . . . . . . . . . . . . . 183

8.2 Other Modeling Approaches . . . . . . . . . . . . . 183

8.3 Model Development . . . . . . . . . . . . . . . . . . . 185

8.4 Normal-Like-or-Better Performance . . . . . . . . . . . . . . . 191

8.5 Discussion . . . . . . . . . . . . . . . . . . . 195

8.5.1 Weights Flexibility . . . . . . . . . . . . . 195

8.5 .2 Longitudinal Data . . . . . . . . . . . . . . 196

8.5.3 The Single Driver and Single Cost Pool Case . . . . 196

8.5.4 Limitations and Further Research . . . . . . . . . 196

8.6 Some Final Remarks on the Single Cost Pool Case . . . . . . 197

8.7 Benchmark Cost Matrices . . . . . . . . . . . . . . . . . . . 198

8.7.1 Technically Efficient Benchmark Cost Matrices . . . . 200

8.7.2 The Target Linear Programming Model . . . . . . . . 200

8.8 MPE Estimation of the Objective Coefficient Vector . . . . . 201

8.9 A Likelihood Model for the Data . . . . . . . . . . . . . . 203

8.9.1 Density and Likelihood Models . . . . . . . . . . . 204

8.10 Genetic Search Space and Technical Issues . . . . . . . . . . . . 205

8.10.1 Representation of the A-Matrices . . . . . . . 205

8.10 .2 Extreme Points and Set Volumes . . . . . . . . . 206

8.10.3 Genetic Search for All Parameters . . . . . . . . . 207

8.11 Validation Issues . . . . . . . . . . . . . . . . 207

8.11.1 Use of the Target-Mode Agreement Criterion . . . . 207

8.11.2 The Comparison to Regression Coefficients Criterion . . 208

8.12 Miscellaneous Issues . . . . . . . . . . . . . . . . 208

8.12 .1 Data Requirements Issues . . . . . . . . . . . . 208

8.12 .2 The Independence Assumption . . . . . . . . . . . . 209

8.12 .3 Marginal Costs . . . . . . . . . . . . . . . . . 209 
8.13 Modification of Assumptions . . . . . . . . . . 209

8.13.1 Limiting Drivers to Historical Ranges . . . . . . . . 209

8.13.2 Discretionary Cost Pool Allocations . . . . . . . . . 210

8.13.3 Department-Specific Output Priorities . . . . . . . . . 210

8.13.4 Department-Specific Performance Densities . . . . . . 211

8.14 Conclusion and Extensions . . . . . . . . . . . 211

Chapter 9 Open Questions and Future Research 213

9.1 Benchmark Cost Matrices . . . . . . . . . . . . . . . . 214

9.2 Chaos . . . . . . . . . . . . . . . . . . 214

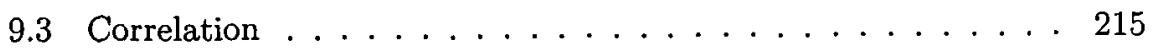

9.4 MDR, MLE and Related Estimation Issues . . . . . . 216

9.5 Probability (Frequency) Moments and Related Integrals . . . 219

9.6 Simulation. . . . . . . . . . . . . . . . . . . . . . . . . . . 220

9.7 Tolstoy's Law . . . . . . . . . . . . . . . . . . . 221

9.8 Unimodality . . . . . . . . . . . . . . . . 221

Bibliography 223

List of Tables 235

List of Figures $\quad 237$

List of Notations $\quad 239$

Author Index 241

Subject Index 245 